\title{
EVOLUÇÃO DA CARDIOPATIA CHAGÁSICA CRÔNICA I - INFLUÊNCIA DA PARASITEMIA
}

\author{
José Borges Pereira, Henry Percy Faraco Wilcox e José Rodrigues Coura
}

\begin{abstract}
No periodo de 8 anos (1982-1990) foi avaliada a evolução da cardiopatia chagásica crônica (CCC), pelo estudo eletrocardiográfico de repouso, eda parasitemia peloxenodiagnóstico em 279 pacientes, 85 homens e 194 mulheres, com idades de 7 a 76 anos (média $=42,6$ anos), todos do municipios de Virgem da Lapa, Estado de Minas Gerais, Brasil. De acordo com os resultados dos eletrocardiogramas classificamos a evolução da CCC em inalterada (EI) quando não havia mudança no padrão inicial do traçado, progressiva (EP) - quando havia mudança de normal para alterado ou pelo agravamento das alterações e regressiva (ER) quando havia normalização ou redução da gravidade das alterações. De acordo com os resultados dosxenodiagnósticos (minimo de 3 e máximo de 8 por paciente), 120 pacientes foram considerados com parasitemia positiva - um ou mais exames positivos e 159 com parasitemia negativa - todos os exames negativos. Os resultados mostraram: a) EI em 172 (61,6\%) pacientes, EP em 99 (35,5\%) e ER em 8 (2,9\%), sem diferença significativa entre o tipo de evolução e o tipo de parasitemia; b) a EP foi crescente com a idade tanto no grupo com parasitemia positiva como no grupo com parasitemia negativa e significativamente maior nos homens em relação às mulheres, independentemente do tipo de parasitemia. Com estes achados podemos afirmar que o aparecimento ou a progressão da CCC não se mostraram associadas ao tipo de parasitemia, mas sim ao sexo masculino e ao aumento da idade dos pacientes, sugerindo que a parasitemia náo está relacionada com o agravamento da cardiopatia chagásica crônica.
\end{abstract}

Palavras-chaves: Doença de Chagas. Cardiopatia chagásica crônica. Estudo longitudinal. Parasitemia. Xenodiagnóstico.

Os estudos longitudinais sobre a doença de Chagas crônica (DCC), desenvolvidos principalmente emáreas encémicas do Brasil como Bambuí-MG ${ }^{9}$, São Felipe-BA ${ }^{13}$, Virgem da Lapa$\mathrm{MG}^{20}$, Pains e Iguatama- $\mathrm{MG}^{8}$, Sul do Rio Grande do Sul-RS ${ }^{2}$, Castro Alves-BA ${ }^{15}$ e Sertão do Estado da Paraíba-PB ${ }^{19}$, ou em áreas da Venezuela como Carabobo ${ }^{16}$, Belén e Eneal ${ }^{22}$, têm mostrado o caráter progressivo da $\mathrm{CCC}$ em importante parcela dos pacientes, enquanto outros permanecem com suas formas clínicas inalteradas por anos, décadas ou mesmo por toda a vida. $E$ até o momento não há explicações consistentes que justifiquem essa diferença de comportamento, embora algumas hipóteses procurem associá-la a diferentes fatores ligados à amostra infectante do T. cruzi às condições sócio-econômicas do paciente

Instituto Oswaldo Cruz/FIOCRUZ, Rio de Janeiro, RJ, Brasil Endereço para correspondência: Dr. José Borges Pereira. Depto. de Medicina Tropical/FIOCRUZ. CP: 926- 20001970 Rio de Janeiro, RJ.

Recebido para publicação em 19/09/91. ou a sua resposta imune na interação com este parasito.

No elenco de fatores ligados ao T.cruzi $e$ ao hospedeiro destacamos a parasitemia que, na fase crônica da infecção, pode ser detectada em até $50 \%$ dos pacientes através de xenodiagnósticos ${ }^{6}{ }^{21}$, permitindo, com isto, a realização de estudos comparativos entre grupos com parasitemia positiva e negativa. Foi com base nestas observações que propusemos a presente investigação, tendo como objetivo principal a tentativa de identificação de uma possível associação entre a progressão da $\mathrm{CCC}$ e a presença ou não de parasitos circulantes (parasitemia).

\section{MATERIAL E MÉTODOS}

Pacientes. Em janeiro de 1982 foi composta uma amostra aleatória simples de 343 pacientes chagásicos crônicos, 137 homens e 206 mulheres, com idades de 7 a 76 anos (média de 41,5 anos), 
Pereira JB, Willcox HPF, Coura JR. Evolução da cardiopatia chagásica crônica. I-Influência da parasitemia. Revista da Sociedade Brasileira de Medicina Tropical 25:101-108, abr-jun, 1992.
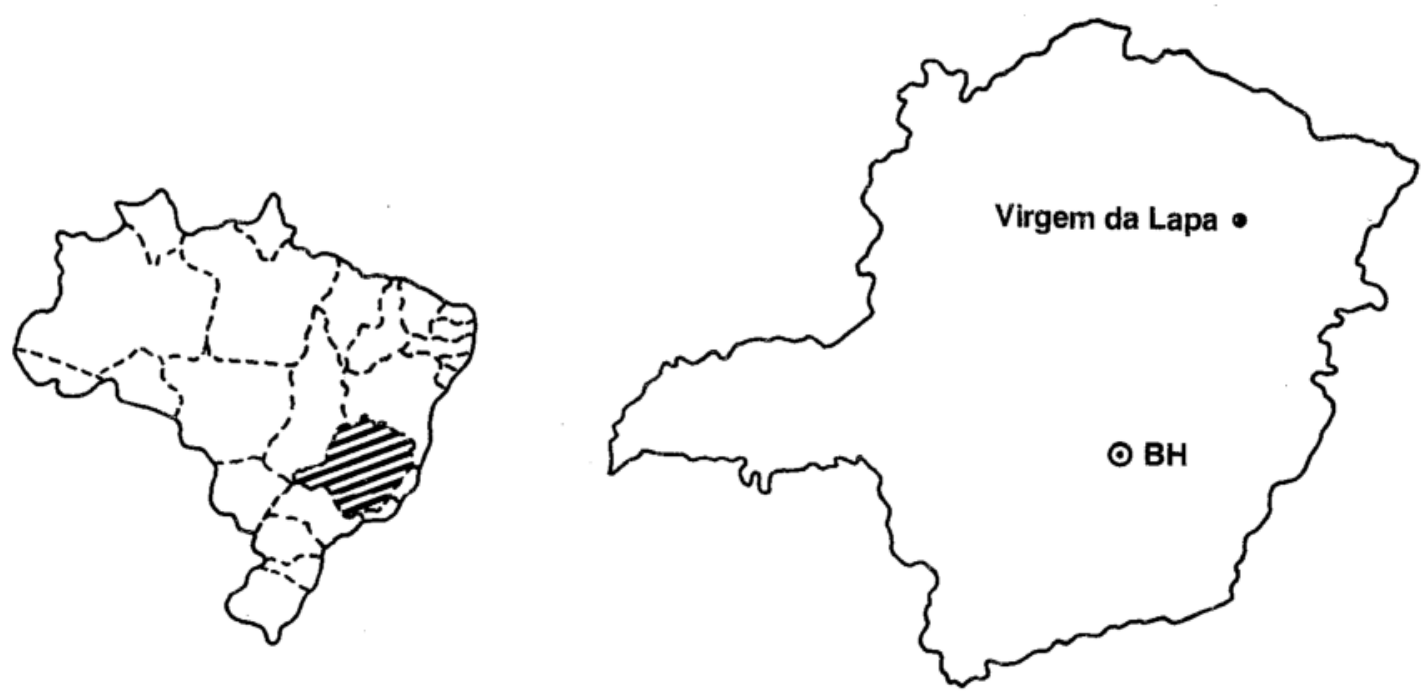

Figura 1 - Brasil, Estado de Minas Gerais - Localização da área estudada (Virgem da Lapa).

correspondente a $25 \%$ da população chagásica estudada no município de Virgem da Lapa, Vale do Jequitinhonha, Estado de Minas Gerais, Brasil (Figura 1). Oito anos depois (janeiro de 1990) restavam na área 279 pacientes, 85 homens e 194 mulheres que integram este trabalho. Dos demais 64 pacientes (52 homens e 12 mulheres), 42 migraram e 22 morreram neste intervalo de tempo. Todos os pacientes, em etapa anterior, haviam sido caracterizados como chagásicos crônicos através da soropositividade para IgG anti T.cruzi pelos testes de imunofluorecência indireta (IFI), hemaglutinação e fixação do complemento, além da soronegatividade para IgM contra o mesmo parasito pelo teste de IFI.

Parasitemia. Foi definida a partir dos resultados dos xenodiagnósticos aplicados, mínimo de 3 e máximo de 8 por paciente, durante o tempo do estudo. Consideramos parasitemia positiva $(\mathrm{Pp})$ o resultado de um ou mais exames positivos $e$ parasitemia negativa $(\mathrm{Pn})$ quando todos os exames eram negativos. Com estes critérios, 120 pacientes foram classificados como portadores de $P$ p e 159 de $\mathrm{Pn}$, distribuídos por faixa etária e sexo na Tabela 1. Cada xenodiagnóstico aplicado continha 40 ninfas

Tabela 1 - Distribuição dos pacientes chagásicos crônicos estudados.

\begin{tabular}{cccccccc}
\hline \multirow{2}{*}{$\begin{array}{l}\text { Faixa } \\
\text { etária }\end{array}$} & \multicolumn{2}{c}{ Homens } & & \multicolumn{2}{c}{ Mulheres } & & \multicolumn{2}{c}{ Total } \\
\cline { 2 - 7 } & $\mathrm{Pp}$ & $\mathrm{Pn}$ & & $\mathrm{Pp}$ & $\mathrm{Pn}$ & & $\mathrm{Pn}$ \\
\hline $07-19$ & 8 & 8 & 14 & 12 & 22 & 20 \\
$20-39$ & 9 & 14 & 34 & 44 & 43 & 58 \\
$40-59$ & 17 & 21 & 24 & 46 & 41 & 67 \\
$>60$ & 4 & 4 & 10 & 10 & 14 & 14 \\
\hline Total & 38 & 47 & 82 & 112 & 120 & 159 \\
\hline
\end{tabular}

$\mathrm{Pp}=$ parasitemia positiva; $\mathrm{Pn}=$ parasitemia negativa. 
Pereira JB, Willcox HPF, Coura JR. Evolução da cardiopatia chagásica crônica. I-Influência da parasitemia. Revista da Sociedade Brasileira de Medicina Tropical 25:101-108, abr-jun, 1992.

de $3^{\circ}$ e/ou $4^{\circ}$ estadios de Triatoma infestans e/ou de Panstrogylus megistus, para um repasto de 30 minutos. A leitura do xenodiagnóstico foi feita pelo exame de cada ninfa 45 dias depois do repasto, através da observação microscópica das fezes obtidas por compressão abdominal ou de todo o tubo digestivo triturado em PBS 7.2, nos casos de fezes negativas.

Evolução da cardiopatia. Foi avaliada pelo estudo eletrocardiográfico. De cada paciente foi obtido o mínimo de dois ECG em repouso, respectivamente de janeiro de 1982 e de 1990. A obtenção e a leitura dos traçados foram feitas pelas mesmas pessoas. Em cada ECG foi obtido o registro das doze derivações clássicas com o mínimo de 3 complexos por derivação e D2 longo nos casos de arritmias. A interpretação foi feita de acordo com as recomendações da New York Heart Association (NYHA). Os resultados dos dois exames, no intervalo de 8 anos, nos permitiram classificar a evolução da CCC em: inalterada (EI), quando não havia mudança no padrão inicial do ECG; progressiva (EP), quando havia mudança de normal para anormal ou pelo agravamento das alterações e regressiva (ER), quando havia normalização do traçado ou redução da gravidade das alterações.

Hipótese e análise dos dados. Admitimos como hipótese de trabalho que a frequência da evolução progressiva da CCC seria maior no grupo de pacientes com parasitemia positiva. Para testá-la empregamos o qui-quadrado $\left(\mathrm{X}^{2}\right)$ considerando nível de significância para $p=0,05$.

\section{RESULTADOS}

De acordo com o expresso na Tabela 2, as frequências de EI, EP e ER não mostraram diferença significativa ( $p>0,05)$ ao compararmos os grupos Pp e Pn. No global, 35,5\% dos pacientes apresentaram EP, nos permitindo estimar a média anual efetiva (não linear) de progressão da CCC em $3,8 \%$.

Em ambos os grupos Pp e Pn, a EP da CCC aumentou com a idade dos pacientes (Figura 2), ressaltando-se que, na faixa de 40 a 59 anos, a frequência de EP foi significativamente maior no grupo $\mathrm{Pp}$ em relação ao grupo $\mathrm{Pn}$.

A frequência de EP da $\mathrm{CCC}$ foi significativamente maior nos homens em comparação com as mulheres, independentemente do tipo de parasitemia (Tabela 3).

Não houve diferença significativa entre o número de casos novos de CCC nos grupos $\mathrm{Pp}$ $(19: 65=29,3 \%)$ e Pn $(28: 86=32,6 \%)$, do mesmo modo que o observado para os casos antigos que apresentaram agravamento $(25: 55=45,4 \%$ no grupo $P$ e e $27: 73=37,0 \%$ no grupo $P n$ ).

$\mathrm{Na}$ Tabela 4 estão assinaladas as frequências das alterações eletrocardiográficas que surgiram e que regrediram. Destacam-se, com maiores incidências, as alterações primárias da repolarização ventricular e as extra-sístoles ventriculares em ambos os grupos Pp e Pn, além da exclusiva incidência de BAV no grupo Pn.

Tabela 2 - Evolução da cardiopatia chagásica crônica de acordo com a parasitemia.

\begin{tabular}{lcccccccc}
\hline Parasitemia & $\begin{array}{c}\text { Pacientes } \\
\text { examinados }\end{array}$ & $\mathrm{n}^{\text {o }}$ & $\%$ & & \multicolumn{2}{c}{ EP } & & ER \\
\hline Positiva & 120 & 74 & 61,7 & 44 & 36,7 & 2 & 1,7 \\
Negativa & 159 & 98 & 61,6 & 55 & 34,6 & 6 & 3,8 \\
\hline Total & 279 & 172 & 61,6 & 99 & 35,5 & 8 & 2,9 \\
\hline
\end{tabular}

$E I=$ evolução inalterada; $E P=$ evolução progressiva; $E R=$ evolução regressiva . 
PereiraJB, Willcox HPF, Coura JR. Evolução da cardiopatia chagásica crônica. I-Influência da parasitemia. Revista da Sociedade Brasileira de Medicina Tropical 25:101-108, abr-jun, 1992.

\section{(\%) PERCENTUAL}

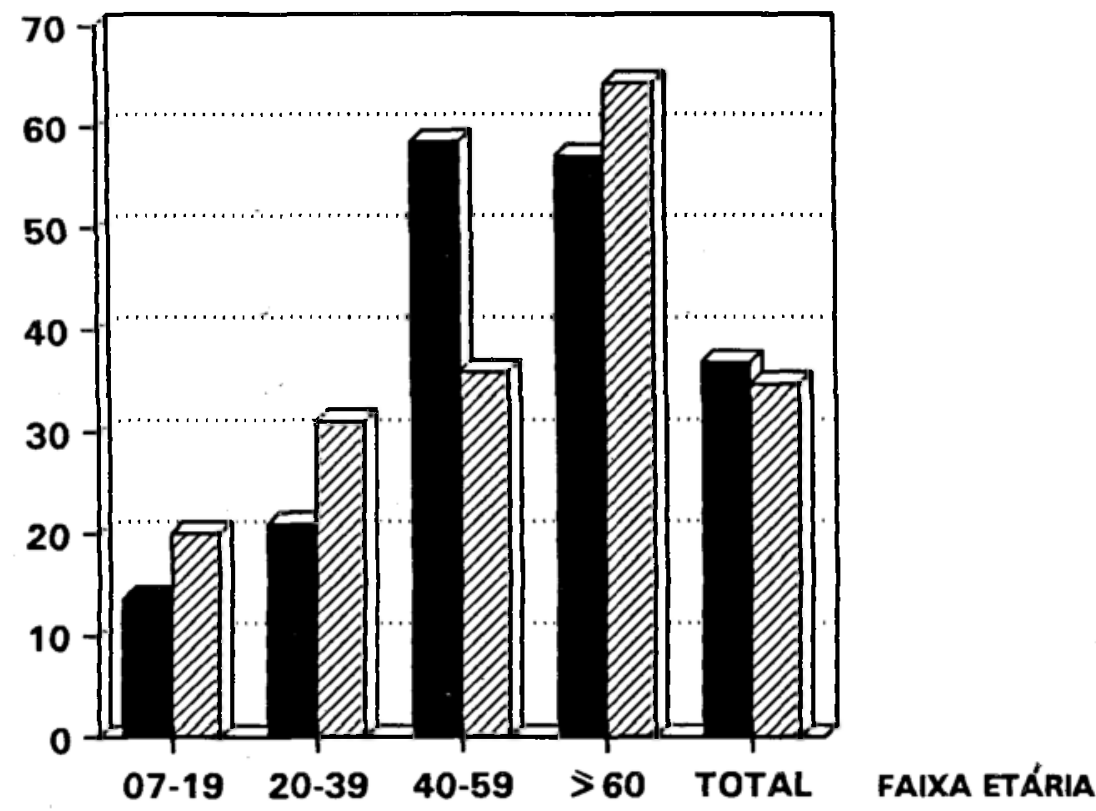

$\begin{array}{lccccc}\text { PACIENTES Pp } & 13.6 & 20.9 & 58.5 & 57.1 & 36.7 \\ \text { PACIENTES Pn } & 20 & 31 & 35.8 & 64.3 & 34.6\end{array}$

\section{PACIENTES Pp PAD PACIENTES Pn}

Fïgura 2 - Evolução progressiva da cardiopatia chagásica crônica de acordo com a faixa etária e a parasitemia.

\section{DISCUSSÃO}

A nossa hipótese de que a progressão da CCC seria maior no grupo de pacientes com parasitemia positiva fundamentou-se nas observações de diversos autores ${ }^{3522}$ que assinalam a presença de "nichos parasitários"no miocárdio de chagásicos crônicos, embora em menor quantidade do que na fase aguda. Estes achados nos permitiram admitir a possibilidade de multiplicação dos parasitos, gerando com isto a

Tabela 3 - Evolução progressiva (EP) da cardiopatia chagásica crônica de acordo com a parasitemia e o sexo.

\begin{tabular}{|c|c|c|c|c|c|c|c|}
\hline \multirow[t]{2}{*}{ Parasitemia } & \multicolumn{3}{|c|}{ Homens } & \multicolumn{3}{|c|}{ Mulheres } & \multirow[t]{2}{*}{$X^{2}$} \\
\hline & $\mathrm{n}^{0}$ & EP & $\%$ & $\mathrm{n}^{\circ}$ & EP & $\%$ & \\
\hline Positiva (Pp) & 38 & 21 & 55,3 & 82 & 23 & 28,0 & $8,2(\mathrm{~S})$ \\
\hline Negativa (Pn) & 47 & 23 & 48,9 & 112 & 32 & 28,6 & $6,0(\mathrm{~S})$ \\
\hline Total & 85 & 44 & 51,8 & 194 & 55 & 28,3 & $14,1(S)$ \\
\hline
\end{tabular}

(S) = significativo 
Pereira JB, Willcox HPF, Coura JR. Evolução da cardiopatia chagásica crônica. I-Influência da parasitemia. Revista da Sociedade Brasileira de Medicina Tropical 25:101-108, abr-jun, 1992.

Tabela 4 - Alteraf̧ões eletrocardiogräficas que incidiram e que regrediram de acordo com a parasitemia.

\begin{tabular}{|c|c|c|c|}
\hline Alterações que incidiram & $\mathrm{Pp}$ & Pn & Total \\
\hline Alteração primária da repolarização ventricular & 14 & 19 & 33 \\
\hline Extra-sístoles ventriculares (*) & 12 & 11 & 23 \\
\hline Hemibloqueio ventricular esquerdo & 7 & 5 & 12 \\
\hline Sobrecarga ventricular esquerda & 2 & 10 & 12 \\
\hline Bloqueio AV I, II e III & 0 & 9 & 9 \\
\hline Bloqueio completo do ramo direito - BRD III & 3 & 5 & 8 \\
\hline Bradicardia sinusal & 6 & 2 & 8 \\
\hline Sobrecarga auricular esquerda & 4 & 3 & 7 \\
\hline Bloqueio incompleto do ramo direito - BRD I & 2 & 2 & 4 \\
\hline Fibrilação auricular & 1 & 0 & 1 \\
\hline Zona eletricamente inativa & 0 & 1 & 1 \\
\hline Baixa voltagem do QRS & 1 & 0 & 1 \\
\hline Bloqueio incompleto do ramo esquerdo - BRE I & 1 & 0 & 1 \\
\hline \multicolumn{4}{|l|}{ Alterações que regrediram } \\
\hline Extra-sístoles ventriculares & 1 & 5 & 6 \\
\hline Bloqueio AV I & 1 & 1 & 2 \\
\hline Bradicardia sinusal & 0 & 1 & 1 \\
\hline Extra-sístoles supraventricular & 0 & 1 & 1 \\
\hline
\end{tabular}

$\mathrm{Pp}=$ parasitemia positiva; $\mathrm{Pn}=$ parasitemia negativa

$(*)$ incluem as polimórficas, bigeminadas e em salvas

ruptura de células e a liberação de $T$. cruzi para a circulação sanguínea, expressando-se, respectivamente, pelo aumento de lesão do miocárdio e pela parasitemia que podem ser detectados através do exame eletrocardiográfico e do xenodiagnóstico. No entanto, os resultados obtidos indicaram independência entre a progressão da CCC e o tipo de parasitemia, conduzindo à rejeição desta hipótese, à semelhança das observações feitas por Castro ${ }^{6}$ em pacientes crônicos de Mambai-GO, nos quais, em estudo seccional (único dessa natureza encontradona literatura, até o momento), não foi observada influência da parasitemia no quadro clínico da DCC.

Considerando os limites da metodologia empregada neste estudo, os resultados nos permitem levantar questão sobre que papel desempenha o $T$. cruzi circulante na evolução da DCC. Se desempenha, quais são os mecanismos? Ação direta? Indução da resposta imune do hospedeiro? Ambos ou outros mecanismos? Quaisquer respostas encontradas contribuirão de maneira siginificativa no entendimento da história natural da infecção chagásica e na conduta terapêutica específica e inespecífica dos pacientes, sobretudo no sentido de 
que eles obtenham reais benefícios ao serem submetidos a estes tipos de tratamento. Dentro desta abordagem sobre que vantagem obtém o paciente chagásico crônico ao ser submetido ao tratamento específico, Macedo e Silveira ${ }^{14}$ não observaram diferença significativa entre as frequências de progressão da DCC (forma indeterminada) nos grupos de pacientes tratados $\mathrm{e}$ não-tratados, no período de 7 anos. Este estudo nos coloca diante da necessidade de publicações que considerem as investigações longitudinais sobre a infeç̧ão chagásica crônica em grupo de pacientes curados em comparação com grupo de pacientes não-tratados. Tais informações poderão definir a importância da presença do $T$. cruzi e seus produtos antigênicos no processo evolutivo da doença crônica.

A análise específica dos resultados deste trabalho mostra que a progressão da CCC, carcterizada pelos casos novos e o agravamento dos casos antigos, em $35 \%$ dos pacientes, com uma taxa média anual efetiva de $3,8 \%$, está de acordo com as taxas encontradas em outras áreas endêmicas de Minas Gerais $^{8}$ e Bahia $^{13}{ }^{15}$ porém se mostra superior às taxas encontradas em estudos realizados no Rio Grande do Sul ${ }^{2}$ e no Sertão da Paraíba ${ }^{19}$, indicando para estas regiões, provavelmente, menor patogenicidade na interação T.cruzi- homem.

O aumento da frequência da EP com a idade dos pacientes revela a importância do tempo de infecção no desenvolvimento da doença na fase crônica. Acreditamos que outros fatores etiopatogênicos da miocardiopatia nos idosos possam estar influindo no incremento da EP da CCC com a idade, tendo em vista que estudos com pareamento de chagásicos e não-chagásicos ${ }^{10} 18$ têm mostrado que a proporção do componente etiológico exclusivamente chagásico na cardiopatia aumenta até a quinta década da vida, decrescendo nas décadas seguintes.

Os homens apresentaram maior frequência de progressão da CCC do que as mulheres, independentemente do tipo de parasitemia (Tabela 3). Não existem explicações com base em invetigações sistematizadas para este achado, embora no campo das idéias se possa admitir que fatores biogenéticos, sócio-culturais e relacionados ao trabalho ligados ao sexo masculino exerçam maior influência no desenvolvimento da miocardiopatia chagásica crônica.

Conforme os estudos longitudinais citados neste texto, as alterações ventriculares e as extra-sístoles ventriculares foram as anormalidades que mais incidiram, marcando assim a progressão da CCC. Entre os possiveis fatos que podem determinar o aparecimento destas alterações, na DCC, podemos citar a hipóxia celular decorrente da vasculopatia ${ }^{411} 17$ ou a lesão da célula miocárdica pela citotoxidade ${ }^{723}$. Ressalta-se que ambos os mecanismos podem ocorrer independentemente do parasitismo tissular, fundamentando as idéias daqueles que acreditam ser prescindível a presença do parasito na patogenia na DCC, a partir de determinado momento, ainda não definido, da infecção, além de consubstanciar os resultados dos estudos epidemiológicos que não observam influências da parasitemia no processo evolutivo da doença crônica.

Apesar de considerarmos o tipo regressivo de evolução, por não encontrarmos outro termo que expresse adequadamente a dinâmica eletrocardiográfica da $\mathrm{CCC}$, da qual pode resultar a normalização e a redução do nível de alterações, não acreditamos na regressão efetiva das lesões miocárdicas, na DCC, sem terapêutica. Acreditamos que a possível mudança no padrạ̃o histológico do miocárdio, no decorrer do processo evolutivo, gere mudanças eletrofisiológicas que permitam as flutuações nos traçados eletrocardiográficos.

\section{SUMMARY}

During eight years (1982-1990) the evolution of chronic chagasic cardiopathy and its relation to parasitemia was evaluated in 279 patientes, 85 men and 194 women, studied by resting eletrocardiography and xenodiagnosis. All patients were residents in Virgem da Lapa, State of Minas Gerais, Brazil and their ages varied from 7 to 76 years (average 42.6y). According to the results of the electrocardiograms the evolution of chagasic cardiopathy was classified as a) unchanged - when there was no change of the initial pattern off the ECG, b) progessive - when there was deterioration of theECG pattern and c) regressive - when there was normalization or regression of the ECG alterations. Regarding xenodiagnosis 120 were considered with positive parasitemia, one or more xenodiagnoses positive, and 159 with negative parasitemia - all xenodiagnoses negative. The results showed: a) chagasic cardiopathy unchanged in $172(61.6 \%)$ patients, b) progressive in 99 (35.5\%) patients and c) regressive in $8(2.9 \%)$. There was no relation between the evolution of chagasic 
Pereira JB, Willcox HPF, Coura JR. Evolução da cardiopatia chagásica crônica. I-Influência da parasitemia. Revista da Sociedade Brasileira de Medicina Tropical 25:101-108, abr-jun, 1992.

cardiopathy and parasitemia. Independent from parasitemia, the cardiopathy was progressive according to the age of the patients and significantly greater in males. In conclusion we can state that evolution of chronic chagasic cardiopathy is associated with the age and with the male sex, but not with parasitemia, and this may suggest that parasitemia is not related to the development of the chronic chagasic cardiopathy.

Key-words: Chagas'disease. Chronic chagasic cardiopathy. Longitudinal study. Parasitemia. Xenodiagnosis.

\section{AGRADECIMENTOS}

Ao Dr. Leônidas Deane pela revisão do texto e sugestões. Ao Dr. Wille Oigman pela colaboração na leitura dos ECG. A Dra. Angela Cristina Junqueira e aos técnicos Júlio Cesar Miguel, Nelmo Bruno, Joaquim Mendes e Zulmira Rios pela participação na leitura dos xenodiagnósticos. A Mozar José Coelho pela incansável colaboração na etapa de campo. Ao Dr. Nelson Chagas pela orientação na análise estatística dos dados.

\section{REFERÊNCIAS BIBLIOGRÁFICAS}

1. Acosta AM, Santos-Buch CA, Autoimmune myocarditis induced by Trypanosoma cruzi. Circulation 71: 1255-1261, 1985.

2. Alcantara A, Baruffa G, Aquino Neto JO, Olintho A, Savoldi T, Lassen C. Epidemiologia da doença de Chagas no RS - Revisão de pacientes após 10 anos de evolução. I-Metodologia. II Grupo chagásico. III-Grupo controle IM-Anais do XIX Congresso da Sociedade Brasileira de Medicina Tropical, Rio de Janeiro p.35-37, 1983.

3. Andrade ZA. Anatomia patológica da doença de Chagas. Revista Goiana de Medicina 4:103-119, 1958.

4. Andrade ZA, Castro Filho BG. As lesões vasculares na miocardite crônica chagásica. Gazeta Médica da Bahia 70:105-112, 1970.

5. Andrade ZA, Ramalho LMP. Miocardite chagásica (Estudo morfológico de 38 casos comprovados pelo encontro de parasitos nas secções histológicas). Gazeta Médica da Bahia 66:55-67, 1966.

6. Castro $\mathrm{CN}$. Influência da parasitemia no quadro clínico da doença de Chagas crônica. Revista de Patologia Tropical 9: 73-136, 1980.

7. Cossio PM, Damilano G, Vega MT, Laguens RP, Mecjert PC, Diez C, Arana RM. In vitro interaction between lymphocytes of chagasic individuals and heart tissue. Medicina (Bueno Aires), 36:287-293, 1976.

8. Coura JR, Abreu LL, Pereira JB, Wilcox HPF. Morbidade da doença de Chagas. Estudo longitudinal de dez anos em Pains e Iguatama, Minas Gerais, Brasil. Memórias do Instituto Oswaldo Cruz 80: 7380,1985

9. Dias JCP. Doença de Chagas em Bambuí, Minas Gerais, Brasil. Estudo clínico-epidemiológico a partir de fase aguda, entre $1940 \mathrm{e} 1982$. Tese de doutourado, Universidade Federal de Minas Gerais, Belo Horizonte, 1982.
10. Dubois LE. Morbidade da doença de Chagas. Estudo seccional em uma área endêmica. Tese de mestrado, Universidade Federal do Rio de Janeiro, Rio de Janeiro, 1977.

11. Laranja FS, Dias E, Nobrega GC, Miranda A. Chagas'disease. A clinical, epidemiologic and pathologic study. Circulation 14: 1035-1060, 1956.

12. Lopes ER, Chapadeiro E, Almeida HO, Rocha A. Contribuição ao estudo da anatomia patológica dos corações de chagásicos falecidos subitamente. Revista da Sociedade Brasileira de Medicina Tropical 9:269-282, 1975

13. Macedo VO. Influência da exposição à reinfecção na evolução da doença de Chagas. Estudo longitudinal de cinco anos. Tese de mestrado, Universidade Federal do Rio de Janeiro, Rio de Janeiro, 1973.

14. Macedo VO, Silveira CA. Perspectivas da terapêutica específica na doença de Chagas. Ëxperiências na forma indeterminada". Revista da Sociedade Brasileira de Medicina Tropical 20 (Supl II): p. M24, 1987.

15. Maguire $J H$, Mott KE, Hoff $R$, Guimarães $A$, França JT, Souza JAA, Ramos WB, Sherlock I. A three-year follow-up study of infection with Trypanosoma cruzi and electrocardiographic abnormalities ina rural community in Northeast Brazil. American Journal of Tropical Medicine and Hygiene 31: 42-47, 1982.

16. Moleiro F, Pifano F, Anselmi G, Ruesta A. La dinamica epidemiologica de la enfermedad de Chagas en el Valle de los Naranjos, Estado Carabobo, Venezuela. III - Evaluation longitudinal del dano miocardio en casos de enfermedad de Chagas en fase crónica del Valle de los Naranjos. Estado Carabobo, Venezuela, Archivos Venezuelano de Medicina Tropical y Parasitologia 5: 47-83, 1973.

17. Okumura M, Correa Neto A, Sila AC. Contribuição para o estudo da patogenia das lesões vasculares na 
Pereira JB, Willcox HPF, Coura JR. Evolução da cardiopatia chagásica crônica. I-Influência da parasitemia. Revista da Sociedade Brasileira de Medicina Tropical 25:101-108, abr-jun, 1992.

doença de Chagas experimental em camundongos brancos. Revista Paulista de Medicina 61: 265-266, 1962.

18. Pereira JB, Coura JR. Morbidade da coença de Chagas. Estudo seccional em uma área endêmica, Virgem da Lapa, Minas. Revista da Sociedade Brasileira de Medicina Tropical 19: 139-148, 1986.

19. Pereira Jb, Cunha RV, Wilcox HPF, Coura JR. Evolução da Cardiopatia chagásica crônica humana no Sertão do Estado da Paraiba, Brasil, no período de 4,5 anos. Revista da Sociedade Brasileira de Medicina Tropical 23: 141-147, 1990

20. Pereira JB, Willcox HPF, Coura JR. Morbidade da coença de Chagas. Estudo longitudinal de seis anos, Virgem de Lapa, Minas Gerais, Brasil. Memórias do Instituto Oswaldo Cruz 80: 63-71, 1985.

21. Pereira JB, Willcox HPF, Marcondes CB, Coura
JR. Parasitemia em pacientes chagásicos crônicos avaliada pelo índice de triatomíneos infectados no xenodiagnóstico. Revista da Sociedade Brasileira de Medicina Tropical 22: 39-44, 1989.

22. Puigbó JJ, Rhde JRN, Barrios HG, Yepez CG, Cuatro años de estudio longitudinal de una communidad rural con endemecidad chagasica. Boletim de la Oficina Sanitaria Panamericana 66:112$120,1969$.

23. Santos-Buch CA, Teixeira ARL. The immunology of experimental Chagas'disease. III. Rejection of allogenic heart cells in vitro. Journal of Experimental Medicine 140: 38-53, 1974.

24. Torres $\mathrm{CM}$. Sobre a anatomia patológica da doença de Chagas. Memórias do Instituto Oswaldo Cruz 36: 391-404, 1941. 